\title{
CHROMATOGRAPHIC BEHAVIOUR AND ANALYTICAL METHOD DEVELOPMENT FOR METFORMIN HCL: APPLICATION TO PERMEATION STUDIES THROUGH CACO-2 CELLS
}

\author{
IMAD I. HAMDAN ${ }^{1 *}$, DUA'A G. FARAH ${ }^{2}$ and RANA ABU-DAHAB ${ }^{1}$ \\ ${ }^{1}$ School of Pharmacy, The University of Jordan, Amman, Jordan \\ ${ }^{2}$ Faculty of Pharmacy, Al Zarqa University, Al Zarqa, Jordan
}

\begin{abstract}
Metformin $\mathrm{HCl}(\mathrm{Mtf})$ is a polar compound with low bioavailability. Counter ions have been shown to improve the bioavailability of polar ionizable drugs. The goal of this work was to develop an HPLC method that is capable of separating and quantifying Mtf from a group of selected organic anions: diclofenac sodium (DS), citric acid (CA), hydroxyl cinnamic acid (HCA), 8-anilinonaphthalene-1-sulfonic acid (ANS), and trisodium phosphate (TSP). Thus, the effect of the mixture of anions on the transport of Mtf through Caco-2 cells could be studied. During the development of the method, interesting chromatographic behaviors of Mtf were observed using a polar stationary phase $\left(\right.$ Hypersil ${ }^{\circledR}$ SAS, C1). The developed method was validated and found to be linear in the range of $2-100 \mu \mathrm{g} / \mathrm{mL}$ with good accuracy and precision. The method was applied for transport experiments of $\mathrm{Mtf}$ across Caco-2 cells in the presence and absence of organic anions. Apparent permeability coefficients (Papp) were calculated. The Papp of Mtf was increased in the presence of CA and DS from $3.37 \times 10^{-6} \mathrm{~cm} / \mathrm{s}$ to $5.08 \times 10^{-6}$ and $4.25 \times 10^{-6} \mathrm{~cm} / \mathrm{s}$ respectively, while it was decreased to $1.9 \times 10^{-6} \mathrm{~cm} / \mathrm{s}$ (p-value 0.01) in the presence of HCA. Interestingly, the Papp for Mtf increased more than four-fold when present with both calcium and CA together, which might lead to significant improvement in the bioavailability of the drug.
\end{abstract}

Keywords: Metformin, Caco-2 cells, ion pairs, polar compounds, permeability

Metformin (Mtf) belongs to the class of drugs known as biguanides and is widely used for the treatment of Type II diabetes (1). It is highly soluble in water and has two pKa values (2.8 and 11.5) so that it exists largely as a hydrophilic cation at physiologic $\mathrm{pH}$ values (Fig. 1). The molecule retains hydrophilicity even in its unionized form (log P-value -1.43). As a result of these physicochemical properties, Mtf is classified as a class III drug according to the biopharmaceutical classification system (2). Owing to its inherent hydrophilicity, Mtf is only moderately absorbed with oral bioavailability of 50$60 \%$. Intestinal absorption of $\mathrm{Mtf}$ is believed to be a result of different mechanisms including passive diffusion (3), paracellular (substantial) and, to some extent, carrier-mediated transport (4-5). Despite its popularity, the use of Mtf is impaired by its moderate absorption and uncomfortable gastrointestinal adverse effects (1). Some efforts have been made to improve the bioavailability of Mtf including the synthesis of several prodrug derivatives (6).
Ion pairing is a technique that has been used to improve the permeability of molecules containing ionizable groups by adding an oppositely charged species to the ionized polar drug, leading to the formation of a more lipophilic ion-pair complex, where the charges are neutralized, and the resultant more lipophilic complex can cross the lipid bilayer (7). Examples of improving intestinal absorption of ionizable polar drugs through ion pairing techniques include indomethacin, trospium chloride, amifostine, oseltamivir, and zanamivir (8-11).

Therefore, Mtf is an ideal candidate for ion-pairing with an acidic counterion. In this study, a group of organic anions with various degrees of polarity was chosen as model potential ion-pairing agents with $\mathrm{Mtf}$ including diclofenac sodium (DS), p- hydroxycinnamic acid (HCA), 8-anilinonaphthalene-1-sulfonic acid (ANS), citric acid (CA), and trisodium phosphate (TSP) to assess if they might influence the intestinal absorption of Mtf. The selected counterions were chosen based on the criteria of having different

* Corresponding author: e-mail: I.hamdan@ju.edu.jo 
structures and physicochemical properties including acidity, molecular weight, physiological charge, solubility, and lipophilicity, thus creating an opportunity to understand the correlation (if any) between the structure of the anion and its effect on absorption. The physicochemical properties and structures of different acidic counterions are shown in Figure 1.

A human colon adenocarcinoma cell line (Caco2) model has been the most extensively used model in evaluating drug permeability as it shows a good correlation with human intestinal absorption (12-13). Thus, these cells have been employed in this study to assess the permeation of Mtf in the absence and presence of the selected organic anions, separately and as mixtures. A reliable chromatographic method was needed in order to quantitatively determine $\mathrm{Mtf}$ in the presence of a mixture of selected hydrophilic and lipophilic anions. Determination of the polar Mtf on the most frequently used HPLC columns (C18) has been a challenge on its own because the highly polar drug has no sufficient retention on the lipophilic C18 stationary phase (14-15). Previously reported methods attempted to overcome the high polarity of Mtf using techniques such as the addition of ion-pair forming agents to the mobile phase where the supposedly formed ion-pairs would enhance the retention of $\mathrm{Mtf}$ on the non-polar columns (16-17).

The purpose of this work was to develop a simple and valid HPLC method for the separation and quantification of $\mathrm{Mtf}$ in Caco-2 cell culture medium in the presence of the selected hydrophilic and lipophilic anions. The developed method, which revealed some interesting chromatographic behavior of $\mathrm{Mtf}$ on a rather polar column, was applied for studying the effect of different anions on the permeation of Mtf.

\section{EXPERIMENTAL}

\section{Material and equipment}

Mtf HCL and DS were kind gifts from al Taqaddom Pharmaceutical IND and JPM respectively. ANS and CA were from Fluka Chemicals (Switzerland) and Panreac Quimica (Barcelona, Spain) respectively. An American type culture collection of Caco-2 cell line (ATCC) and all other chemicals were from Sigma, USA. Penicillin at 10.000 units $/ \mathrm{mL}$, streptomycin at $10.000 \mu \mathrm{g} / \mathrm{mL}$, gentamicin at $10 \mathrm{mg} / \mathrm{mL}$, and glutamine at 29.2 $\mathrm{mg} / \mathrm{mL}$ solution $100 \mathrm{X}$ were from GE Healthcare HyClone $^{\circledast}$, USA. Dulbecco's modified Eagle's medium, Trypsin/EDTA solution (10X), and fetal bovine serum (FBS) were from Biochrome, Germany. Phosphate buffer saline (PBS) and Trypan blue $0.5 \%$ were from Biowest (France) and Euroclone (Italy). A 3-(4,5-dimethylthiazoyl)-2,5diphenyltetrazolium bromide (MTT) cell proliferation assay kit was from iNtRON Biotechnology, Inc. (Korea).

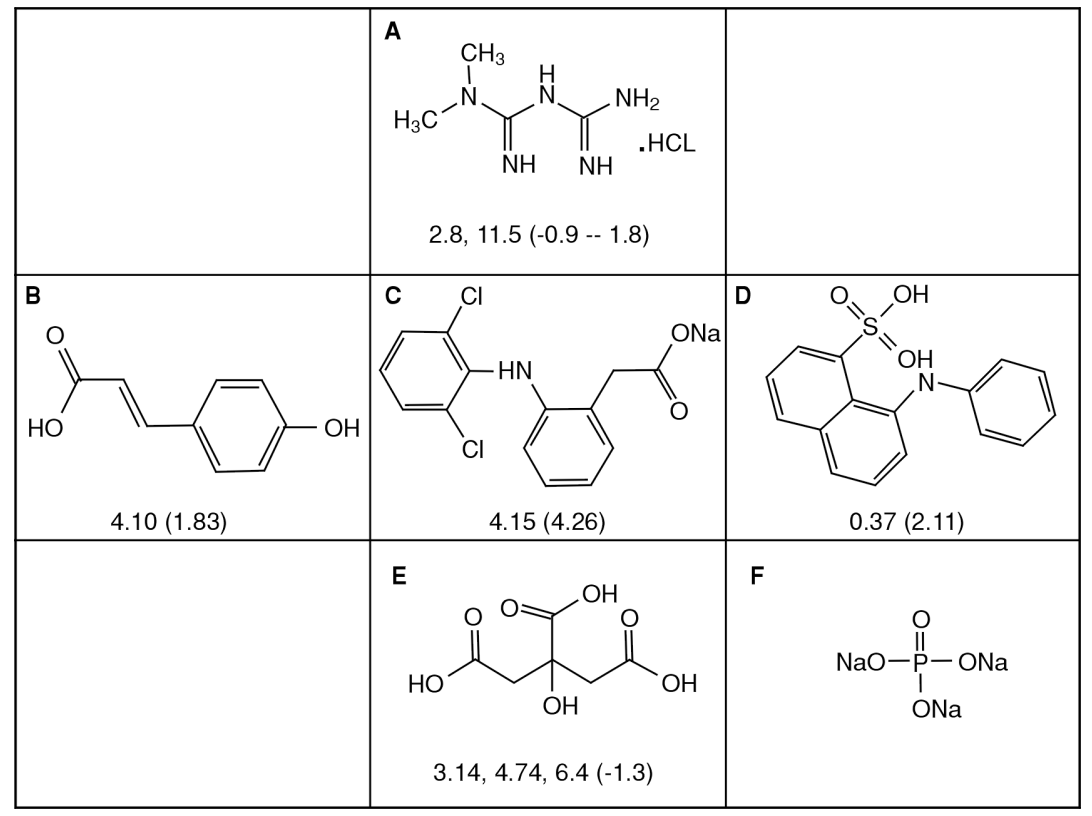

Figure 1. Chemical structures of Mtf (A), HCA (B), DS (C), ANS (D), CA (E), and TSP (F) together with their reported pka, in bold, and $\log \mathrm{P}$ values in parenthesis. 
The employed HPLC consisted of a pump (LC20AT), a UV/Vis detector (SPD-20A UV/VIS), and a column oven (CTO-20A), which were all from Shimadzu (Japan).

Initially, a standard C18 column was tested but eventually, the more polar SAS column (Hypersil $^{\mathrm{TM}}$ SAS C1; $250 \mathrm{~mm} \mathrm{~L} \times 4.6 \mathrm{~mm}$ i.d. and $5 \mu \mathrm{m}$ particle size) from Thermo Scientific (USA) was employed. The employed $\mathrm{CO}_{2}$ incubator was from Binder $\mathrm{GmbH}$ (Germany). The epithelial volt/ohm meter (EVOM) was from World Precision Instruments, Inc. (USA). The absorbance microplate reader and FL $\times 800$ fluorescence microplate reader were from Bio-Tek Instruments, Inc. Transwell inserts ( $12 \mathrm{~mm}$ diameter, $0.4 \mu \mathrm{m}$ pore size) were from Corning Inc., Costar $^{\circledR}$ (USA).

\section{HPLC analysis}

Initially, several mobile phases were attempted in the course of the method development, and these included $90 \%, 40 \%, 30 \%$, and $20 \%$ methanol in 25 $\mathrm{mM}$ sodium phosphate buffer $(\mathrm{pH} 6)$. The finally adopted one consisted of $30 \%$ methanol and $70 \%$ of $25 \mathrm{mM}$ sodium phosphate buffer with $\mathrm{pH}$ adjusted to 6 . The mobile phase was pumped in an isocratic manner at a flow rate of $1 \mathrm{~mL} / \mathrm{min}$. The temperature of the column was maintained at $40^{\circ} \mathrm{C}$ with $\mathrm{UV}$ detection at $230 \mathrm{~nm}$. All mobile phases were filtered under vacuum through a $0.45 \mu \mathrm{m}$ nylon filter, and all injection volumes were kept at $20 \mu \mathrm{L}$.

\section{Preparation of standard solutions}

A standard stock solution of Mtf was $200 \mu \mathrm{g} / \mathrm{mL}$ and prepared in phosphate-buffered saline (PBS). DS,

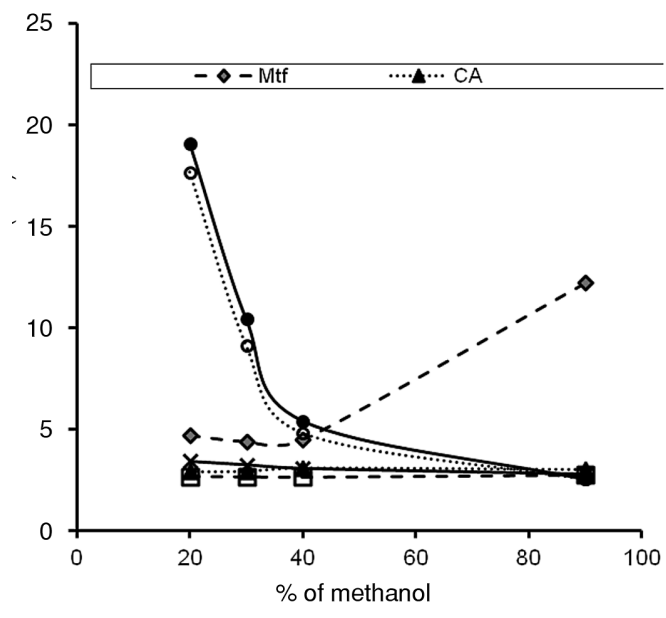

Figure 2. Relationship between retention time in minutes and percentage of methanol in mobile phase using SAS column at $\mathrm{pH}$ 6.0. Note that the very hydrophilic analytes (CA, HCA and TSP) almost coeluted without significant retentions at all percentages.
ANS, and HCA standard stock solutions of 200 $\mu \mathrm{g} / \mathrm{mL}$ were prepared using a mixture of methanol and water $(50: 50)$. A citric acid standard stock solution of $1,000 \mu \mathrm{g} / \mathrm{mL}$ was prepared in methanol and water $(50: 50)$ and a trisodium phosphate standard stock solution of $3000 \mu \mathrm{g} / \mathrm{mL}$ in a PBS buffer.

The working standard solutions of the organic anions (DS, ANS, and HCA) were prepared with concentrations of 20 and $100 \mu \mathrm{g} / \mathrm{mL}$. For citric acid, the resulted final concentrations were 100 and $500 \mu \mathrm{g} / \mathrm{mL}$. Solutions with a maximum soluble concentration of each anion were prepared, and then the mixtures were prepared by adding a $0.5 \mathrm{~mL}$ aliquot of each anion solution in a $20 \mathrm{~mL}$ volumetric flask and a $0.5 \mathrm{~mL}$ aliquot of metformin solution of $1.0 \mathrm{mg} / \mathrm{mL}$. Finally, the volume was increased to $20 \mathrm{~mL}$ using PBS.

\section{Method validation}

The developed method was validated in terms of specificity, linearity, accuracy, limit of detection, limit of quantification, intra-day precision, and repeatability of measurement.

In order to investigate the specificity of the method, chromatograms were obtained for Mtf dissolved in PBS as well as each organic anion dissolved in PBS, PBS alone, cell culture blank (Hank's buffered salt solution [HBSS] from basolateral compartments) and Mtf organic anion mixtures. For the evaluation of linearity, standard solutions were prepared at eight different concentration levels over a range of $2-200 \mu \mathrm{g} / \mathrm{mL}$ in the matrix of the cellular diffusion experiment. The calibration curve was constructed by plotting the peak area against the concentrations of Mtf. Three calibration curves were constructed, and linearity was judged by examining the determination coefficient $\left(r^{2}\right)$ and y-intercept of the linear regression line for each calibration curve. The data obtained during the linearity study was used to assess the working concentration range.

The accuracy of the method was determined by subjecting three standard solutions with known concentrations of Mtf, namely 30, 50, and $90 \mu \mathrm{g} / \mathrm{mL}$, to the proposed HPLC method. All concentrations were within the linear range and were injected in triplicates. Repeatability (intra-day precision) was determined by assaying ten replicate injections of an Mtf solution of $90 \mu \mathrm{g} / \mathrm{mL}$ in the same day under the same experimental conditions. Results were expressed as relative standard deviations (RSD\%).

Limits of detection (LOD) and quantification (LOQ)were determined based on the standard deviation of the response and the slope of the calibration curve, according to the following equations respectively: $\mathrm{LOD}=3.3 \sigma / \mathrm{S}$ and $\mathrm{LOQ}=10 \sigma / \mathrm{S}$ where $\sigma$ 
is the standard deviation of responses (Y-intercept) and $\mathrm{S}$ is the slope of the calibration curve.

\section{Cell culture}

Determination of maximum nontoxic concentration

Caco- 2 cells were thawed and maintained as an attached monolayer cell culture in Dulbecco's Modified Eagle Medium (DMEM), supplemented with $10 \%(\mathrm{v} / \mathrm{v})$ heat-inactivated FBS, $1 \%$ (v/v) penicillin-streptomycin, and $0.14 \%(\mathrm{v} / \mathrm{v})$ gentamicin. Caco-2 cells were grown in T-75 flasks, then incubated at $37^{\circ} \mathrm{C}$ in an atmosphere of $5 \% \mathrm{CO}_{2}$, and routinely maintained by replacing the culture media with a fresh one every 2-3 days and by regular passage.

In order to determine Mtf's and each organic anion's maximum non-toxic concentration that could be used on Caco- 2 cells, an MTT proliferation assay was performed. In summary, Caco- 2 cells at a density of $1 \times 10^{4}$ cells per well were seeded on 96well plates and incubated for 10 days until confluent monolayers were obtained. On the day of the experiment, the cell culture media was removed, and monolayers were washed three times and incubated with PBS for $2 \mathrm{~h}$ at $37^{\circ} \mathrm{C}$. PBS was aspirated, and solutions of $\mathrm{Mtf}$ and each organic anion in PBS were added separately to each well (6 serial dilutions at $1: 2$ ratio were used starting from maximum soluble concentration); the cells were then incubated for $1 \mathrm{~h}$. Following incubation, cells were washed with PBS, then $100 \mu \mathrm{L}$ of culture media and $15 \mu \mathrm{L}$ of MTT reagent were added to the wells, and the cells were incubated for an additional $4 \mathrm{~h}$. After the $4 \mathrm{~h}$ incubation, the produced formazan crystals were dissolved by the addition of dimethyl sulfoxide (DMSO) to each well, and the absorbance was measured at $570 \mathrm{~nm}$ using a 96-well plate reader. All of the experiments were conducted in quadruplicate.

\section{In-vitro permeability assay for metformin}

Caco- 2 cells with an average cell density of $1.5 \times 10^{5}$ cells $/ \mathrm{cm}^{2}$ were seeded onto 12-well Transwell plates and allowed to grow for 21 days until confluent monolayers were obtained. Culture media was changed every 2-3 days. Monolayer integrity was measured by recording the transepithelial electrical resistance (TEER) before and after treatment with each drug using an epithelial volt/ohm meter. TEER values were also obtained in the absence of cells and were considered as background (blank). Total TEER values were corrected for background to obtain the $\mathrm{TEER}_{\text {final }}$ value. Membranes were used in the experiments when the TEER final $_{\text {Value reached }} \geq 1000 \Omega . \mathrm{cm}^{2}$. Once the TEER level was established and before the transport study, a Lucifer yellow rejection assay was performed and those inserts that showed rejection higher than $99 \%$ were used.

\section{Apparent permeability coefficient (Papp) of met- formin}

On the day of the experiment, the culture media was first aspirated, and both sides of the monolayers were washed three times with HBSS and preincubated with the transport buffer (HBSS
$\mathrm{mV}$

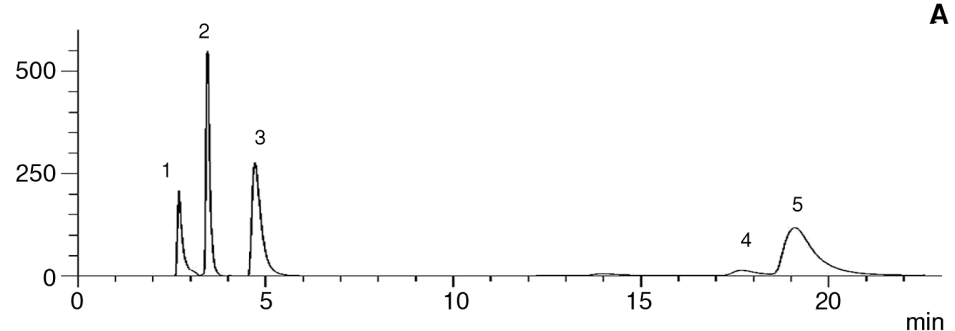

$\mathrm{mV}$

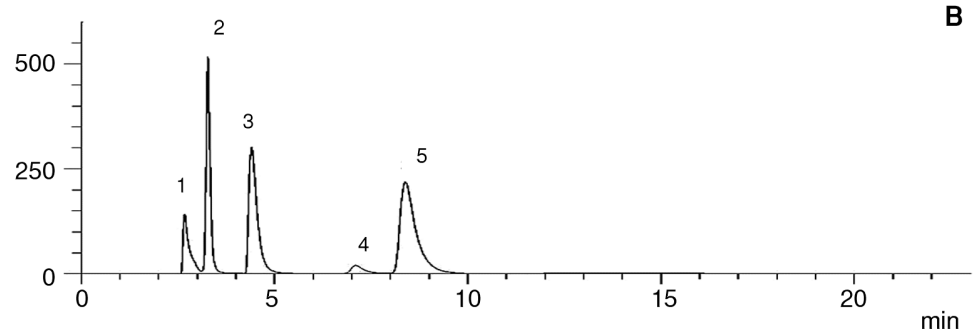

A

Figure 3. HPLC chromatograms of Mtf-anions mixture, using: (A) $20 \%$ and (B) $30 \%$ of methanol in the mobile phase buffered at pH 6 $(1 \mathrm{~mL} / \mathrm{min})$; column temperature $40^{\circ} \mathrm{C}$. Peak identification: $1=\mathrm{TSP} ; 2=\mathrm{HCA} ; 3=\mathrm{Mtf} ; 4=\mathrm{ANS}$ and $5=\mathrm{DS}$. 


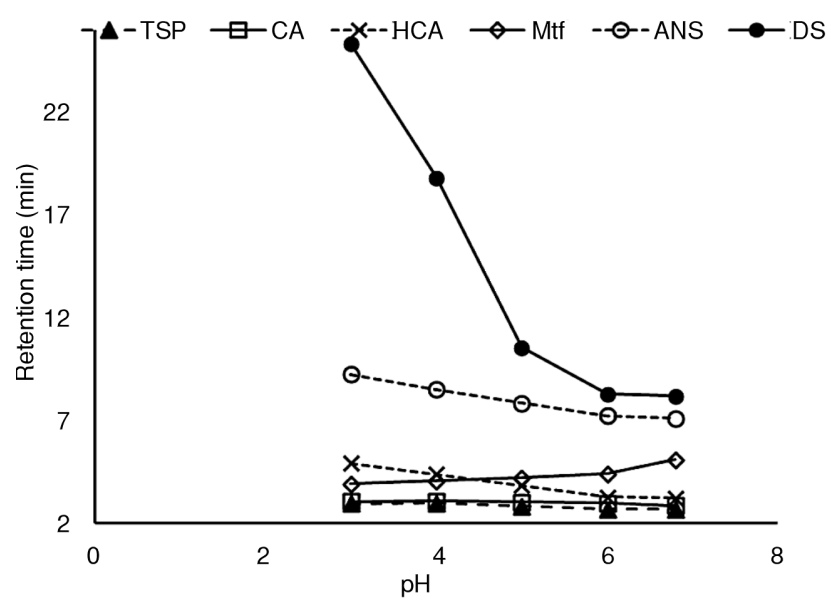

Figure 4. Effect of $\mathrm{pH}$ on the retention times of Mtf and the selected anions.

$\mathrm{pH}=7.4$ ) for $60 \mathrm{~min}$ at $37^{\circ} \mathrm{C}$. Next, $500 \mu \mathrm{L}$ of Mtf solutions alone at $1.0 \mathrm{mg} / \mathrm{mL}$ or in the presence of individual organic anions at their maximum nontoxic concentrations (or mixture of all drugs) were added to the apical compartment (for $\mathrm{A} \rightarrow \mathrm{B}$ transport)while the opposite chambers (basolateral) were filled with $1500 \mu \mathrm{L}$ of fresh HBSS (all experiments were carried out in triplicate). Then, the transport study was initiated by placing the transwell plate on the microplate thermo-shaker incubator at $37^{\circ} \mathrm{C}$ and 250 oscillations per min. Samples $(150 \mu \mathrm{L})$ were taken from the receiver compartments at the time points 30, 60, 120, and $240 \mathrm{~min}$ and were replaced with fresh HBSS to maintain sink conditions. At the last time point $240 \mathrm{~min}$, samples were taken from the donor compartments as well, in order to confirm the mass balance. Finally, the TEER measurement was carried out to reassess Caco- 2 monolayer integrity. Samples were stored at $-20^{\circ} \mathrm{C}$ until the analysis for the Mtf content using HPLC was done.

The transport of Mtf was expressed in terms of the apparent permeability coefficient (Papp) which was calculated using the following equation:

$$
\text { Papp }=\frac{d Q}{d t} \times \frac{1}{C_{0} \times A}
$$

Where: $\mathrm{dQ} / \mathrm{dt}$ is the slope of the linear plot of cumulative amount $(\mu \mathrm{g})$ versus time (second), $\mathrm{C}_{0}$ is the initial concentration of the drug in the donor compartment $(\mu \mathrm{g} / \mathrm{mL})$, and $\mathrm{A}$ is the monolayer growth surface area $\left(1.12 \mathrm{~cm}^{2}\right)$.

\section{RESULTS AND DISCUSSION}

\section{HPLC method development and optimization}

In order to investigate the effect of the selected anions on the permeation of Mtf, it was necessary to develop a simple, valid, and reliable HPLC method that could be applied for separation and quantification of Mtf in the presence of these anions. Although several HPLC methods have been previously reported for the analysis of Mtf, none has dealt with the separation of Mtf from organic anions. Separation of the highly hydrophilic Mtf from other hydrophilic and hydrophobic organic anions on typical reversedphase columns (C18) was quite a challenge and particularly so when a rapid and isocratic system was sought. In this study, after a few preliminary trials with a standard C18 column, it became evident that the separation of the involved compounds would not be possible isocratically due to the very high polarity of Mtf in comparison with the much more lipophilic DS and ANS. Therefore, further trials went on using the rather hydrophilic stationary phase: C1 (Hypersil SAS). Method development started with examining the mobile phases containing decreasing percentages of methanol (90-20\%) in a phosphate buffer $(\mathrm{pH}=6)$. The resultant retention time for each analyte was plotted against the percentage of methanol (Fig. 2). The results confirmed the significantly higher lipophilicity of DS and ANS in comparison to the rest of analytes as they showed vastly longer retention times than those of the rest of the analytes, at low percentages of an organic component (methanol). Other anions, however, showed almost no change in their retention as a function of the percentage of the organic compound which is due to their very low retention. DS and ANS showed a dramatic decrease in retention as the percentage of organic component was increased within the rather narrow range of $20-40 \%$. The retention of $\mathrm{Mtf}$ increased abruptly with an increasing percentage of methanol, at percentages of methanol higher than 
40\%. Therefore, Mtf exhibited a rather uncommon behavior under the conditions of reversed-phase chromatography where the analyte retention time would be anticipated to be inversely proportional to the percentage of organic component. However, such behavior has been previously reported for hydrophilic analytes using typical C18 reversedphase columns (18). It was interesting to note that the inflection point in the profiles of lipophilic anions (DS and ANS) and Mtf was at almost the same range of percentage of the organic component, i.e., $\sim 40 \%$. Thus, we hypothesized that the mechanism of retention on the $\mathrm{C} 1$ column might have been switched from a predominantly reversed-phase mechanism to a rather normal phase one at about that percentage. Overall, the retention time of $\mathrm{Mtf}$ was increased to about $12 \mathrm{~min}$ at high percentage of methanol ( 90\%) which enhanced the possibility of separating Mtf from other polar and non-polar analytes isocratically.

Nevertheless, while the conditions that yielded such long retention times of Mtf ( 90\% methanol) represented good achievements, they were not chosen for further optimization since the peak shape of Mtf was suboptimal in addition to the poorly overlapping peaks of the anionic analytes. The next best condition according to Figure 2 was $30 \%$ of methanol, and thus further optimizations were carried out at that percentage. Due to the very low response of CA (low light absorptivity), it was excluded from further development of the HPLC separation method. Nevertheless, its effect on the permeation of Mtf could still be assessed because it was shown not to interfere with the peaks of other analytes. However, the complete separation of all of the remaining four anions and $\mathrm{Mtf}$ was achieved within a suitable run time ( $9 \mathrm{~min})$ using a ratio of 70 : 30 (25 mM phosphate buffer, $\mathrm{pH}=6$ : methanol) as shown in Figure 3.

While keeping the percentage of methanol constant at $30 \%$, the $\mathrm{pH}$ of the buffer was changed $(3,4$, 5,6 , and 6.8) and chromatograms for the mixture of analytes were obtained. The results are summarized in Figure 4. The retention of Mtf tended to increase, albeit slightly, as the $\mathrm{pH}$ was increased, which was not to be expected for a strongly basic substance such as Mtf, whose degree of ionization is not expected to change within the examined range of $\mathrm{pH}$ (3-6.8) if a typical reversed-phase mechanism was the only acting effect. Therefore, it was the polar interaction, namely with the free residual silanol groups, that was most likely in action. While the examined $\mathrm{pH}$ range was not expected to change the percentage of ionization of Mtf (positively charged at the entire range), the free acidic silanol groups were more likely to be seriously influenced as the pKa values of silanol (3.8-6.8) lay within the examined range (17, 19-20). Thus, when silanol groups became more negatively charged, at higher $\mathrm{pH}$ values, the attraction of the positively charged Mtf was potentiated leading to higher retention. If only typical reversed-phase chemistry and mechanisms were responsible, retardation of $\mathrm{Mtf}$ as a function of increasing $\mathrm{pH}$ (5-6.8) would not be expected because the pKa of $\mathrm{Mtf}$ is far away from the examined range (11.5). Thus, the observed effect pro-

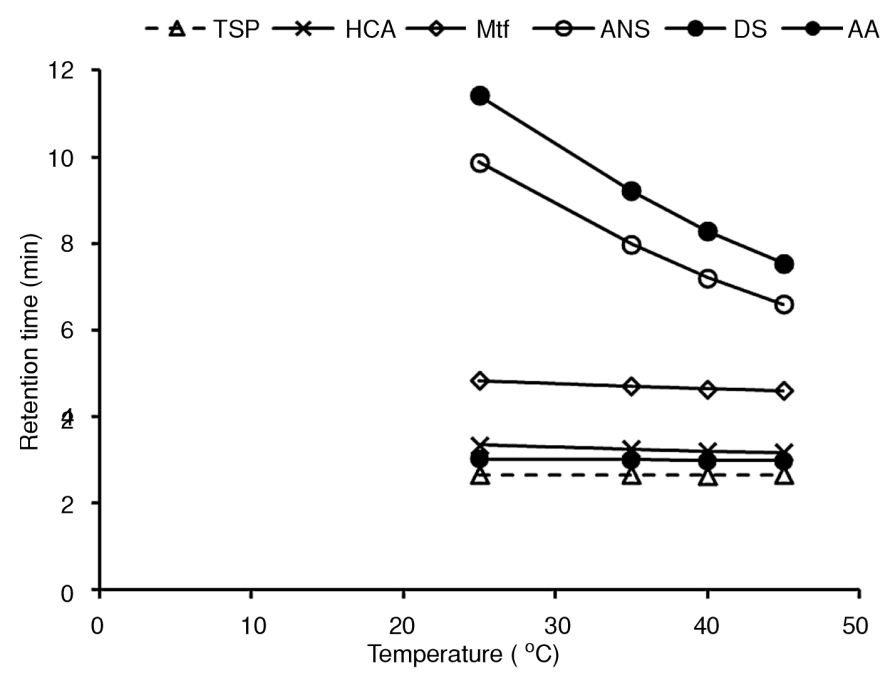

Figure 5. Effect of temperature on retention time of Mtf and the selected anions. 

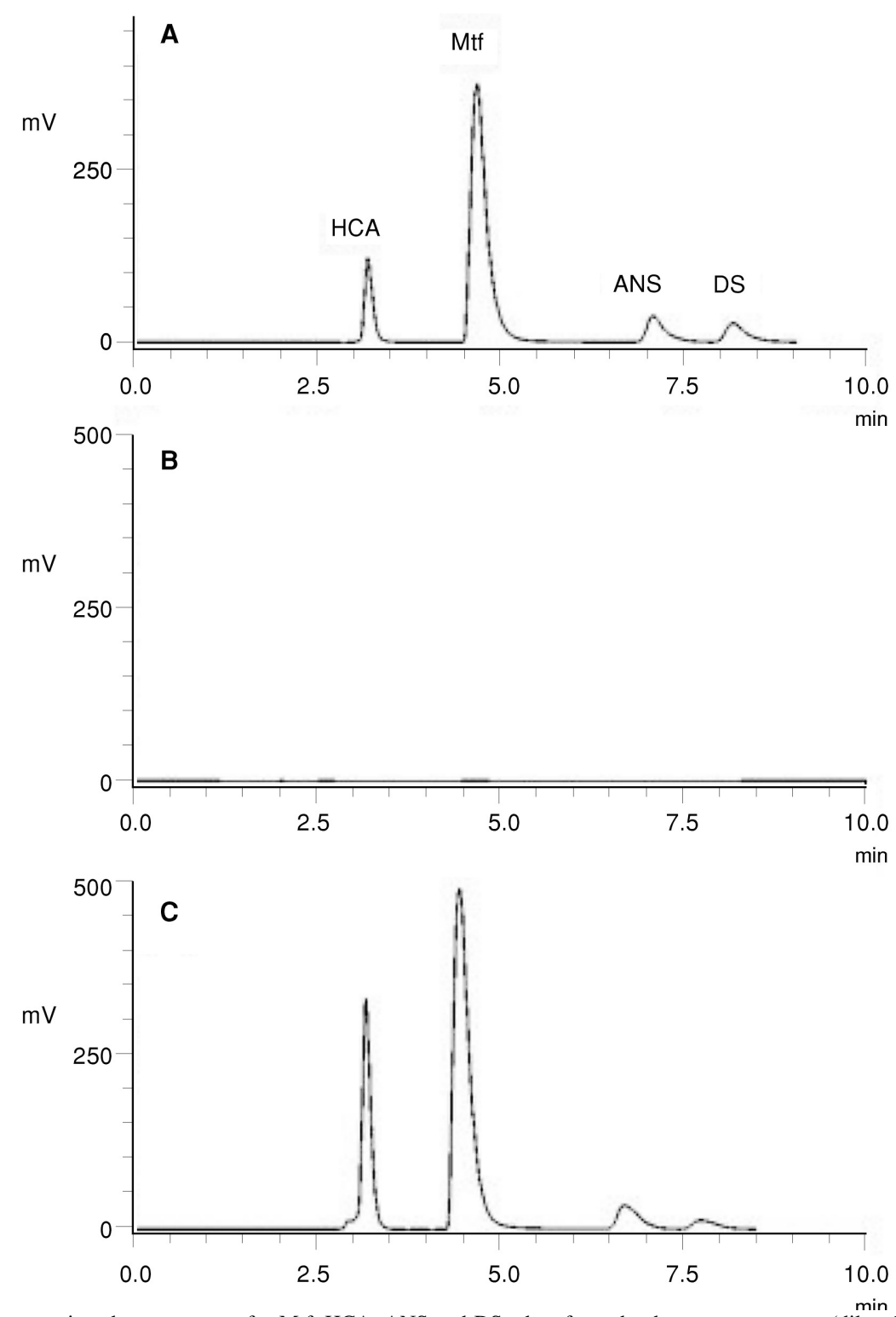

Figure 6. Representative chromatograms for Mtf, HCA, ANS and DS taken from the donor compartment (diluted) at zero time (A), and after $4 \mathrm{~h}$ taken from the receiver compartment (C). Chromatogram in (B) shows the employed incubation matrix. Note how the peak area ratio of $\mathrm{HCA} / \mathrm{Mtf}$ and $\mathrm{ANS} / \mathrm{DS}$ were changed in the receiver compartment as compared to the donor.

vides a direct and perhaps the first evidence that free silanol groups may have (in particular cases such as $\mathrm{Mtf}$ ) the predominant role in $\mathrm{C} 1$ stationary phases.

For organic anions, even more pronounced effects were observed; the retention of the more lipophilic anions decreased significantly when the $\mathrm{pH}$ increased in the range 3 to 6 . The decrease in retention observed at the lower range of $\mathrm{pH}$ (3-6) indicated that it was the increase in the percentage of the ionized form of the anions (carboxylic acids) that led to the decrease of retention. Nevertheless, a per- fect separation of Mtf and baseline separation of the organic anions was achieved at $\mathrm{pH} 6$ and that was the condition recommended for further optimization, validation, and actual application of the method.

While keeping the percentage of methanol constant at $30 \%$ and $\mathrm{pH}$ at 6 , the effect of the column temperature was investigated over the range $25-45^{\circ} \mathrm{C}$, and chromatograms for the mixture of analyte were obtained. The obtained results are presented in Figure 5. Increasing temperatures resulted in shorter retention times, lower backpressures and 
higher peak efficiency. Shorter retention times were obviously noted for the late eluting lipophilic anions compared with the hydrophilic anions (TSP, CA, and AA) which showed nearly constant retention times over the examined range of temperatures. For the late eluting anions, the extent of decrease in retention times was rather typical for reversed-phase columns, i.e., $1-2 \%$ for a temperature increase of $1^{\circ} \mathrm{C}$ (21-22). However, a perfect separation of Mtf and baseline separation of the organic anions within 9 min was achieved at $40^{\circ} \mathrm{C}$, and that was the condition chosen for further validation and actual application of the method.

Different flow rates were tried; increasing the flow rate from $1 \mathrm{~mL} / \mathrm{min}$ to $2 \mathrm{~mL} / \mathrm{min}$ decreased all retention times to about their halves with a concomitant overlapping of some of the anions with
Mtf. Consequently, a flow rate of $1 \mathrm{~mL} / \mathrm{min}$ was chosen to avoid overlap between peaks and to provide a good separation within $9 \mathrm{~min}$.

\section{Method validation}

Method validation was performed according to the International Council on Harmonisation (ICH) guidelines (23). The chromatograms for all organic anions in addition to Mtf were recorded separately under the specifically defined conditions, and the identity of each peak was confirmed by the retention time. Cell culture blanks (transport buffer) were also tested for selectivity by obtaining chromatograms after incubation with cells for $4 \mathrm{~h}$ (Fig. 6) and no co-eluting peaks with Mtf could be detected, which showed that the proposed HPLC method was specific.
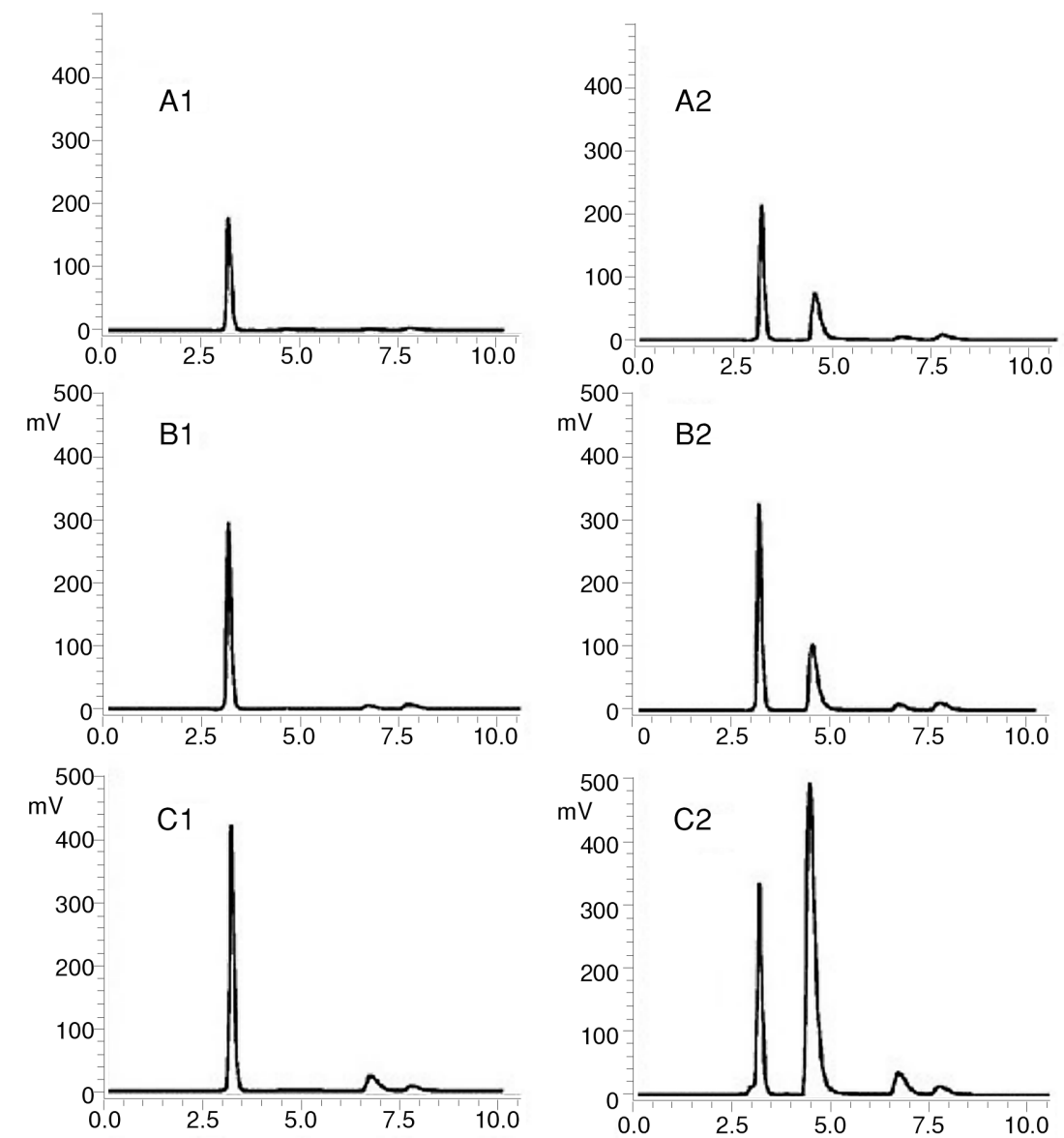

Figure 7. Representative chromatograms for diffusion of a mixture of anions in absence (series 1 to the left) and presence (series 2 to the right ) of Mtf, at time points of 30 (A), 60 (B) and $240 \mathrm{~min}(\mathrm{C})$ of the experiment. Peak labels as in Figure 6A; Note that the peak areas of the anions (ANS and DS) were always larger in Presence of Metf but that for HCA was markedly decreased in presence of Metf at 240 $\min$. 
Table 1. Accuracy results of the concentrations under investigations. Data represent the average and RSD of 3 repetitions.

\begin{tabular}{|c|c|c|c|}
\hline $\begin{array}{c}\text { Added } \\
\text { concentration } \\
(\mu \mathrm{g} / \mathrm{mL})\end{array}$ & $\begin{array}{c}\text { Mean measured } \\
\text { concentration }(\mu \mathrm{g} / \mathrm{mL}) \\
(\mathrm{n}=3)\end{array}$ & RSD\% & \% Accuracy \\
\hline 33 & 33.08 & 1.30 & 100.3 \\
\hline 51.7 & 52.08 & 1.32 & 100.5 \\
\hline 90 & 91.06 & 0.48 & 101.2 \\
\hline
\end{tabular}

Table 2. Apparent permeability coefficient values $(\mathrm{cm} / \mathrm{sec})$ for metformin alone and in presence of each of the organic anions. Each point presents the average determination of 3 wells.

\begin{tabular}{|l|c|c|c|}
\hline & Papp $\left(\times 10^{-6} \mathrm{~cm} / \mathrm{s}\right)$ & SD $(\mathrm{n}=3)$ & p-value \\
\hline $\mathrm{Mtf}$ & 3.37 & $1.83 \mathrm{E}-07$ & \\
\hline $\mathrm{Mtf}+\mathrm{DS}$ & 4.25 & $3.48 \mathrm{E}-07$ & 0.03 \\
\hline $\mathrm{Mtf}+\mathrm{CA}$ & 5.08 & $8.55 \mathrm{E}-07$ & 0.03 \\
\hline $\mathrm{Mtf}+\mathrm{HCA}$ & 1.9 & $1.56 \mathrm{E}-07$ & 0.01 \\
\hline $\mathrm{Mtf}+\mathrm{AA}$ & 2.83 & $1.16 \mathrm{E}-06$ & 0.64 \\
\hline $\mathrm{Mtf}+\mathrm{ANS}$ & 2.85 & $4.94 \mathrm{E}-07$ & 0.44 \\
\hline $\mathrm{Mtf}+\mathrm{CaCl}_{2}+\mathrm{CA}$ & 13.7 & $1.81 \mathrm{E}-06$ & 0.0006 \\
\hline
\end{tabular}

\section{Calibration curve, linearity, and range}

Calibration curves were constructed by plotting the peak area against concentrations of $\mathrm{Mtf}$. Eight concentrations of Mtf were initially used in the range of 2-200 $\mu \mathrm{g} / \mathrm{mL}$, but linearity was only confirmed for the range $2-100 \mu \mathrm{g} / \mathrm{mL}$. Three calibration curves were constructed, and they showed consistent linearity as seen in the consistency of the intercept and correlation coefficient. A typical calibration equation could be given by $\mathrm{y}=136343 \mathrm{x}-$ 17183 with a minimum correlation coefficient of 0.9996 with an RSD for correlation coefficient and slope $=0.021 \%$ and $0.74 \%$ respectively.

For the assessment of accuracy, the ICH guidelines recommend collecting data from a minimum of nine determinations over a minimum of three concentration levels covering the specified range (three concentrations, three replicates each). The mean observed concentrations for all three concentrations levels of 30,50, and 90 $\mu \mathrm{g} / \mathrm{mL}$ were calculated using the resulted regression equation. Then RSD and percent accuracy of the mean concentrations were calculated. The results are shown in Table 1, and it is evident that the method is accurate and precise within the desired range with RSD values less than $1.3 \%$. LOD and LOQ for Mtf were found to be 0.43 $\mu \mathrm{g} / \mathrm{mL}$ and $1.45 \mu \mathrm{g} / \mathrm{mL}$ respectively.

\section{Application to permeation studies}

The calculated maximum non-toxic concentrations were $1,0.75,1.25,0.5$, and $0.5 \mathrm{mg} / \mathrm{mL}$ for $\mathrm{Mtf}$,
DS, CA, ANS, and HCA respectively which confirms the high safety margin of $\mathrm{Mtf}$ and reasonable safety of the anions. TSP, however, was found toxic at all concentration levels tested and thus was not included in further permeation studies. TEER was satisfactory for all inserts before and after the permeation studies. However, Lucifer yellow (LY) rejection assay test values dropped notably only in the wells that contained citric acid along with calcium chloride.

The apparent permeability coefficient (Papp) and efflux ratio (ER) for Mtf were evaluated using the Caco-2 cell monolayer model system. ER was calculated as the ratio of Papp obtained for basolateral to apical direction divided by that for apical to basolateral direction. Under the previously mentioned conditions, the Papp and ER of Mtf were found to be $3.37 \pm 0.018 \times 10^{-6} \mathrm{~cm} / \mathrm{s}$ and $0.95 \pm$ 0.185 respectively which is in good agreement with that previously reported (3). Mtf was very poorly transported through the Caco-2 monolayers. The low permeability coefficient and an efflux ratio < 1.5 indicate that the paracellular pathway is the main permeation route through Caco- 2 cells.

The effect of each selected organic anion on the Papp of Mtf was studied by the addition of each organic anion with an Mtf solution separately or as mixtures to the apical compartment. The maximum non-toxic concentration of each organic anion was used while keeping the concentration of Mtf con- 
stant at $1.0 \mathrm{mg} / \mathrm{mL}$ in all transport studies. The results are summarized in Table 2. Accordingly, ANS appeared not to have any influence on the Papp of Mtf. DS, on the other hand, led to an increase of Mtf Papp from $3.37 \times 10^{-6} \pm 0.01$ to $4.25 \pm 0.03 \times 10$ ${ }^{6} \mathrm{~cm} / \mathrm{s}$ (p-value 0.03 ), which might be attributed to ion-pair formation. This was in agreement with previous reports (24), where the ability of the diclofenac anion to form an ion-pair with organic cations in aqueous solution was assured. The lower molar ratio of DS relative to the Mtf employed (due to solubility constraints) in the permeation experiment is one possible reason behind the little improvement observed in the permeation of $\mathrm{Mtf}$. Because it was possible to monitor the increase in the peak areas of DS and ANS simultaneously with Mtf, it was evident that the presence of Mtf also resulted in some enhancement of the permeability of DS and ANS (Fig. 7).

A more dramatic effect was observed with CA where the Papp for Mtf was increased 1.63 fold (from $3.37 \pm 0.01$ to $5.08 \pm 0.08 \times 10^{-6} \mathrm{~cm} / \mathrm{s}$ [p-value 0.02]) as shown in Table 2. Considering the high hydrophilicity of CA, it is more likely that the observed increase in the permeation of Mtf was a result of the direct effect of CA on the intercellular junction rather than a lipophilic ion pair formation. The direct effect of citric acid on the tight junction was most likely achieved due to the calcium chelating ability of citric acid (25). Some reports have already pointed out that calcium chelation loosens the intercellular tight junction (26). The ability of CA to enhance the permeability of hydrophilic compounds was also reported (27-28). A powerful calcium chelating agent such as citric acid causes calcium depletion, which in turn leads to considerable changes in the cell and opening of the tight junction. In order to confirm the chelation effect of citric acid, calcium chloride was added to a solution containing both Mtf and citric acid in an excess molar ratio (10 $\mathrm{CaCl}_{2}: 1 \mathrm{CA}$ ). We hypothesized that CA would not lead to calcium depletion and subsequent loosening of the junction in the presence of a high concentration of calcium ions, so consequently, the effect on Mtf permeability should disappear. Surprisingly, the presence of a 10 molar equivalent of calcium led to a striking four-fold increase in the Papp of $\mathrm{Mtf}$ $\left(13.7 \times 10^{-6} \mathrm{~cm} / \mathrm{s}\right)$. Results of the \% LY rejection assay after transport experiments indicated an increase in the LY permeability only in those wells containing a high concentration of calcium and citric acid (\% LY rejection $=96 \%)$. Thus, although against expectations, there was good practical evidence that a high concentration of calcium together with citric acid led to significant changes in the tightness of the intercellular junctions making them more permeable.

HCA was distinguished from all other anions where it consistently showed approximately a twofold decrease in the Papp of Mtf (from 3.37 \pm 0.01 to $1.89 \pm 0.01 \times 10^{-6} \mathrm{~cm} / \mathrm{s}$ (p-value 0.01$)$ ) after incubation for $4 \mathrm{~h}$. HCA is a phenolic natural product and along with other polyphenols has been shown to enhance the tight junction of intestinal cells (29) which might explain the observed significant decrease in absorption of Mtf. On the other hand, Mtf appeared to influence the permeation of HCA. At early time points of the permeation experiment, no effect of Mtf on the permeation of HCA was evident although the effect was obvious on DS and ANS. After 3-4 h, Mtf resulted in a dramatic decrease in the permeated amount of HCA through the Caco-2 monolayer (Fig. 7). Being a naturally occurring compound that might be consumed with food, it might be helpful for patients and clinicians to be aware of the potential interaction between Mtf and HCA so that unwanted variations in bioavailability of Mtf might be avoided.

\section{CONCLUSION}

A reliable method for the determination of the hydrophilic Mtf and some selected organic anions has been developed and validated using the rather uncommonly used polar C1 (SAS) column. An interesting chromatographic behavior of Mtf has been observed were its retention on the column switches from decreasing to increasing as a function of the percentage of organic component in the mobile phase. The observed behavior of $\mathrm{Mtf}$ on the polar reversed-phase column (C1 SAS) could most likely be explained as a result of switch in predominant mechanism influencing retention on the column, i.e., normal (with the residual silanol groups) or reversed-phase mechanism (interaction with the more lipophilic $\mathrm{C} 1$ ). That finding could open a new hope for separating highly polar cationic drugs which usually suffer low retention on commonly used reversed-phase columns, e.g., C18.

Application of the method to permeation experiments for Mtf and the selected anions revealed a significant improvement of Mtf permeability in the presence of the lipophilic DS which is most probably a result of an ion pair formation. A powerful and safe calcium chelator such as citric acid in the presence of a high concentration of calcium led to a four-fold increase in the Papp of Mtf which might be a very promising approach in 
improving the bioavailability of Mtf. HCA showed a negative effect on the absorption of Mtf. Thus, different anions appeared to affect the permeation of Mtf in different manners. Further investigations are necessary to confirm the safety and exact mechanism for the enhancement of permeation using high concentrations of calcium ion and citric acid.

\section{Acknowledgment}

The authors would like to thank the Deanship of Scientific Research at the University of Jordan due to their continuous support. Miss Lara Majdalawi is also thanked for her technical assistance.

\section{Conflict of interests}

The authors declare no conflict of interest.

\section{REFERENCES}

1. Drug Information Handbook: A Comprehensive Resource for All Clinicians and Healthcare Professionals, Lacy C.F., 14th edn., Lexi-Comp Inc., USA 2009.

2. Britnall A.E., Clarke G.S: in Analytical Profiles of Drug Substances and Excipients. Brittain H.G. Ed., Vol. 25, pp. 243-293, Elsevier Academic Press, Amsterdam 1998.

3. Nicklin P., Keates A.C., Page T., Bailey C.J.: Intern. J. Pharm. 128, 155 (1996).

4. Zhou M., Xia L., Wang J.: Drug Metabol. Disposition. 35, 1956 (2007).

5. Müller J., Lips K.S., Metzner L., Neubert R.H., Koepsell H., Brandsch M.: Biochem. Pharmacol. 70, 1851 (2005).

6. Huttunen K.M., Mannila A., Laine K., Kemppainen E., Eppanen J.L., et al.: J. Med. Chem. 52, 4142 (2009).

7. Suresh P.K., Paul S.D.: Sierra Leone J. Biomed. Res. 3, 70 (2011).

8. El Shaer A., Hanson P., Mohammed A.R.: Eur. J. Pharm. Sci. 62, 124 (2014).

9. Heinen C.A., Reuss S., Amidon G.L., Langguth P.: Molec. Pharm. 10, 3989 (2013).
10. Samiei N., Mangas-Sanjuan V., GonzálezÁlvarez I., Foroutan M., Shafaati A., et al.: Eur. J. Pharm. Sci. 49, 499 (2013).

11. Miller J.M., Dahan A., Gupta D., Varghese S., Amidon G.L.: Molec. Pharm. 7, 1223 (2010).

12. Hilgers A.R., Conradi R.A., Burton P.S.: Pharm. Res. 7, 902 (1990).

13. Artursson P., Karlsson J.: Biochem. Biophys. Res. Comm. 175, 880 (1991.

14. Hamdan I., Jaber A.B., Abushoffa A.: J. Pharm. Biomed. Anal. 53, 1254 (2010).

15. Yuen K.H., Peh K.K.: J. Chromatogr. B: Biomed. Sci. Applications. 710, 243 (1998).

16. Chhetri H.P., Thapa P., Van Schepdael A.: Saudi Pharm. J. 22, 483 (2014).

17. Zarghi A., Foroutan M., Shafaati A., Khoddam A.: J. Pharm. Biomed. Anal. 31, 197 (2003).

18. Naidong W., Chen Y.-L., Shou W., Jiang X.: J. Pharm. Biomed. Anal. 26, 753 (2001).

19. Herrero-Martinez J.M., Méndez A., Bosch E., Rosés M.: J. Chromatogr. A. 1060, 135 (2004).

20. Ong S., Zhao X., Eisenthal K.B.: Chem. Phys. Letters. 191, 327 (1992).

21. Ahuja S., Dong M.: in Handbook of Pharmaceutical Analysis by HPLC. Elsevier, Academic Press, Amsterdam 2005.

22. Lindsay S., Barnes J.: in High Performance Liquid Chromatography. Wiley, London 1992.

23. Nahler G.: International Conference on Harmonisation (ICH), Dictionary of Pharmaceutical Medicine (2009).

24. Fini A., Fazio G., Gonzalez-Rodriguez M., Cavallari C., Passerini N., Rodriguez L.: Inter. J. Pharm. 187, 163 (1999).

25. Changa D.: J. Amer. Oil Chem. Soc. 60, 618 (1983).

26. Salamat-Miller N., Johnston T.P.: Inter. J. Pharm. 294, 201 (2005).

27. Froment D.P., Molitoris B.A., Buddington B., Miller N., Alfrey A.C.: Kidney Internatinal. 36, 978 (1989) .

28. Okada H., Yamazaki I., Ogawa Y., Hirai S., Yashiki T., Mima H.: J. Pharm. Sci. 71, 1367 (1982).

29. Rogoll D., Bergmann H., Hellenschmidt D., Heinze J., Scheppach W., et al.: J. Appl. Bot. Food Qual. 83, 110 (2012).

(c) 2020 by Polish Pharmaceutical Society. This is an open access article under the CC BY NC license (c) (i) (\$) (http://creativecommons.org/licenses/BY/4.0/). 